\title{
Pseudomelanosis of the stomach and duodenum: an uncommon endoscopic finding
}

Authors

Institutions
M. Thure Caire ${ }^{1}$, Shivam Kalan ${ }^{2}$, Patrick Brady ${ }^{1}$, Jeffrey Gill ${ }^{3}$

${ }^{1}$ University of South Florida Health, Morsani College of Medicine, Department of Gastroenterology

${ }^{2}$ University of South Florida Health, Morsani College of Medicine, Department of Internal Medicine

3 James A. Haley Veterans Hospital, Department of Gastroenterology submitted

4. April 2014

accepted after revision

23. April 2014

\section{Bibliography}

DOI http://dx.doi.org/

10.1055/s-0034-1377175

Published online: 23.6.2014

Endoscopy International Open

2014; 02: E191-E192

(c) Georg Thieme Verlag KG

Stuttgart · New York

E-ISSN 2196-9736

\section{Corresponding author}

M. Thure Caire, MD

USF Health - Gastroenterology

12901 Bruce B Downs Blvd

Tampa, FL 33612

tcaire@gmail.com
A woman (70-years) with a history of iron deficiency anemia and chronic kidney disease presented with two weeks of abdominal pain. Upper endoscopy demonstrated the gastric and duodenal mucosa was black and speckled consistent with diagnosis of pseudomelanosis. Biopsies showed pigment-laden macrophages in the lamina propria, which stained positive for iron and

\section{Introduction}

\section{$\nabla$}

Pseudomelanosis (PM) of the upper gastrointestinal tract is a rare, incidental endoscopic finding characterized by dark pigmented inclusions in macrophages of the lamina propria. Although the etiology is unknown, PM has been associated with the use of medications, including hydralazine, thiazide diuretics, furosemide, beta blockers, and iron supplements. Hypertension, chronic renal disease, gastric hemorrhage, and diabetes mellitus have also been associated with PM. We present the case report of a woman with findings indicative of a diagnosis of PM of the stomach and duodenum. To our knowledge, this woman is the youngest patient (70 years) reported with a diagnosis of PM of the stomach.

\section{Case report}

$\nabla$

A 70-year-old woman with a history of iron deficiency anemia, chronic kidney disease, and peptic ulcer disease presented to a tertiary care facility with two weeks of post-prandial abdominal pain. Her medications included hydralazine, ferrous sulfate, and furosemide. On upper endoscopy, regions of flat, black-speckled, pigmented mucosa were found in the stomach and duodenum ( Fig. 1, Fig.2). Gastric and duodenal mucosal biopsies demonstrated pigment-laden macrophages in the lamina propria ( $\bullet$ Fig. 3). Iron and Masson-Fontana trichrome staining of the muco-
Masson-Fontana trichrome stain consistent with a "melanin-like" pigment. Although an uncommon endoscopic finding, this pigment has been associated with the use of certain medications, antihypertensives and iron supplements, and systemic illnesses, including hypertension, chronic kidney disease, gastric hemorrhage, and diabetes mellitus.

sa were positive, consistent with the presence of a “melanin-like" pigment ( $\bullet$ Fig.4). This confirmed the diagnosis of gastric and duodenal PM.

\section{Discussion \\ $\nabla$}

PM in the upper gastrointestinal tract is a rare endoscopic finding. Duodenal PM was first described by Bisordi and Kleinman in 1976 [1] and gastric PM by Treeprasertuk et al. in 2000 [2]. PM of the upper GI tract can be found incidentally on endoscopy, but most case reports in the literature describe the indication as abdominal pain. Characteristic findings include flat, black-speckled pigmented mucosa. Unlike pseudomelanosis coli, PM in the upper GI tract does not have a known association with anthraquinone containing laxatives. Melanocytes are not typically present in the GI tract, and the hyperpigmentation in PM is caused by the uptake of exogenous substances by macrophages and the formation of pigmented deposits. The most commonly reported medication associated with PM is oral iron, though associations with diuretics and charcoal ingestion have also been reported [3]. As many as $20 \%$ of samples of affected mucosa do not stain for iron [5]. In a review of seventeen adult patients with PM confirmed by histology, the mucosa from each of three patients (18\%) was negative for Perl's iron stain [4]. Although duodenal PM have been found among children, most case reports of PM duodeni are those of older patients [6], and, to our knowl- 


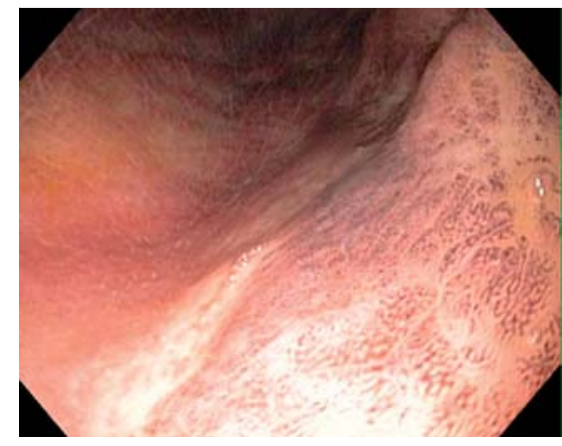

Fig. 1 Stomach with hyperpigmented mucosa.

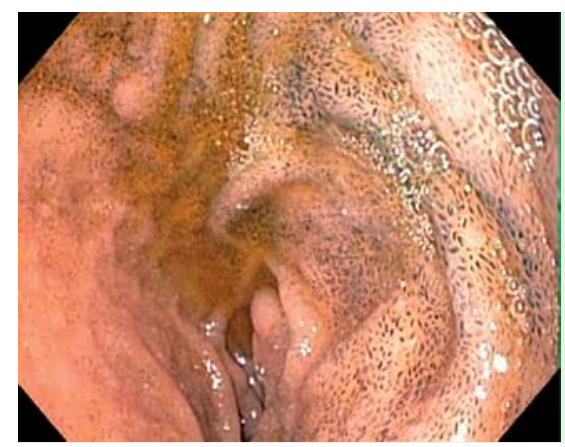

Fig. 2 Duodenum with flat, speckled, hyperpigmented mucosa.

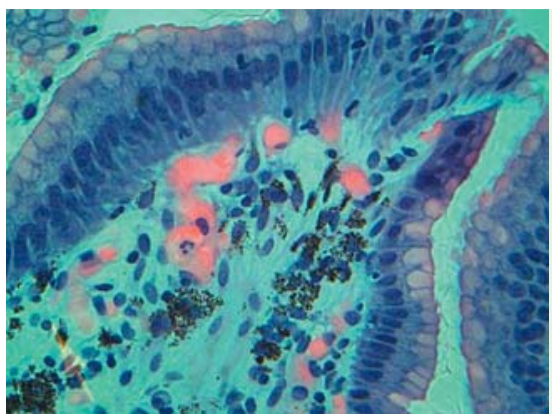

Fig. 3 Gastric mucosa with iron positive, pigment-laden macrophages.

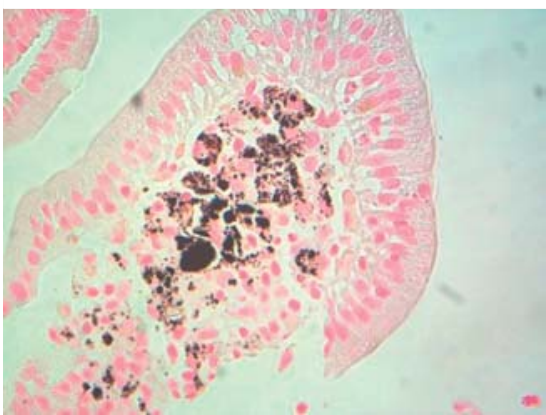

Fig. 4 Masson-Fontana ammoniac silver stained duodenum with a "melanin-like" pigment. edge, the 70-year-old woman described here represents the youngest patient diagnosed with gastric PM reported [2,5,7-9]. Although correlations with drug use (hydralazine, furosemide, hydrochlorothiazide, propranolol, and iron supplements) and systemic diseases (hypertension, chronic renal disease, gastric hemorrhage, and diabetes mellitus) have been noted, the etiology of PM has not been elucidated. Unlike iron deposition elsewhere in the body, which may generate a fibroproliferative response, PM has not been associated with fibrosis or stricture [4]. In addition, there is no evidence that PM is a precursor for neoplasia or any other progressive pathology. Therefore, avoidance of medications associated with the condition should only be recommended if it is felt that the PM is contributing to a specific symptom.

The patient described exhibited findings indicative of PM that included the medical history, medication use, and endoscopic and histological features of the disease. The etiology and significance of PM in the upper GI tract are unknown. However, gastroenterologists may find that upper endoscopy findings consistent with a diagnosis of PM may become more common because of the aging patient population who have been diagnosed with a variety of medical conditions and use numerous medications associated with this diagnosis.

\section{Competing interests: None}

\section{References}

1 Bisordi WM, Kleinman MS. Melanosis Duodeni. Gastrointest Endosc 1976; 23: 37-38

2 Treeprasertuk S, Thong-Ngam D, Suwangool P et al. Pseudomelanosis duodeni: association with hypertension and chronic renal failure: case report. J Med Assoc Thai 2000; 83: 964-968

$3 \mathrm{Kim}$ J, Hwang JK, Choi WS et al. Pseudomelanosis ilei associated with ingestion of charcoal: case report and review of literature. Dig Endosc 2010; 22: $56-58$

4 Giusto D, Jakate S. Pseudomelanosis duodeni: associated with multiple clinical conditions and unpredictable iron stainability - case series. Endoscopy 2008; 40: 165-167

5 Kibria R, Barde CJ. Pseudomelanosis of the Stomach. Endoscopy 2010; 42: E60
6 de Magalhaes Costa MH, Fernandes Pegado Mda G, Vargas C et al. Pseudomelanosis duodeni associated with chronic renal failure. World J Gastroenterol 2012; 18: 1414-1416

7 Weinstock LB, Katzman D, Wang HL. Pseudomelanosis of stomach, duodenum and jejunum. Gastrointest Endosc 2003; 58: 578

8 Pueblitz S, Squires RH, Timmons CF. Pseudomelanosis duodeni in an adolescent male: case report and review of the literature. Pediatr Pathol Lab Med 1997; 17: 115-123

9 Antaki F, Irwin BC, Levi E. Rare occurrence of gastric pseudomelanosis. Gastrointest Endosc 2009; 69: 599 